\title{
Quantitative expression and localization of cysteine and aspartic proteases in human abdominal aortic aneurysms
}

\author{
Fabian Lohoefer ${ }^{1,4}$, Christian Reeps ${ }^{1,4}$, Christina Lipp ${ }^{1}$, Martina Rudelius ${ }^{2}$, Felix Haertl ${ }^{1}$,
} Edouard Matevossian ${ }^{3}$, Alma Zernecke ${ }^{1}$, Hans-Henning Eckstein ${ }^{1}$ and Jaroslav Pelisek ${ }^{1}$

Cysteine and aspartic proteases possess high elastolytic activity and might contribute to the degradation of the abdominal aortic aneurysm (AAA) wall. The aim of this study was to analyze, in detail, the proteases (cathepsins B, D, K, L and S, and inhibitor cystatin C) found in human AAA and healthy aortic tissue samples. The vessel walls from AAA patients $(n=36)$ and nonaneurysmal aortae $(n=10)$ were retrieved using conventional surgical repair and autopsy methods. Serum samples from the same AAA patients and 10 healthy volunteers were also collected. Quantitative expression analyses were performed at the mRNA level using real-time reverse transcriptase-PCR (RT-PCR). Furthermore, analyses at the protein level included western blot and immunoprecipitation analyses. Cellular sources of cysteine/aspartic proteases and cystatin $\mathrm{C}$ were identified by immunohistochemistry (IHC). All cysteine/aspartic proteases and cystatin C were detected in the AAA and control samples. Using quantitative RT-PCR, a significant increase in expression was observed for cathepsins $B(P=0.021)$ and $L(P=0.018)$, compared with the controls. Cathepsin B and cystatin C were also detected in the serum of AAA patients. Using IHC, smooth muscle cells (SMCs) and macrophages were positive for all of the tested cathepsins, as well as cystatin C; in addition, the lymphocytes were mainly positive for cathepsin B, followed by cathepsins D and S. All cysteine/aspartic proteases analyzed in our study were detected in the AAA and healthy aorta. The highest expression was found in macrophages and SMCs. Consequently, cysteine/aspartic proteases might play a substantial role in AAA.

Experimental \& Molecular Medicine (2014) 46, e95; doi:10.1038/emm.2014.20; published online 16 May 2014

Keywords: abdominal aortic aneurysms; cysteine and aspartic proteases; degradation of extracellular matrix

\section{INTRODUCTION}

In the elderly population, abdominal aortic aneurysm (AAA) is a frequent and mostly asymptomatic disease. ${ }^{1}$ Therefore, it often goes undetected until a rupture unexpectedly occurs, resulting in a mortality rate of $>90 \% .^{2-4}$ Thus far and despite all advances in the management of AAA, the only established predictor for the relative, but not individual, risk stratification of asymptomatic AAA is the maximum diameter. ${ }^{5-7}$ Therefore, a better understanding of the pathophysiological processes leading from AAA wall destabilization to rupture may lead to the discovery of new, patient-specific and more accurate predictors of AAA rupture risk. Proteolytic degradation of the extracellular matrix (ECM) is the main factor that leads to AAA wall vulnerability. ${ }^{8}$ Various proteases are involved in this process, particularly the matrix metalloproteases (MMPs). ${ }^{9-11}$ However, the relevance of other proteolytic enzymes is still unclear. Recently, proteases of the cysteine and aspartic family have been proposed as potential contributors of ECM degradation in AAA because of their outstanding collagenolytic and elastolytic potential. ${ }^{11-14}$ Furthermore, it has also been assumed that some cathepsins are involved in the progression of atherosclerosis and neovascularization. ${ }^{14}$

Thus, the aim of the present study was to analyze the expression of the relevant cysteine/aspartic proteases, cathepsins $\mathrm{B}, \mathrm{D}, \mathrm{K}, \mathrm{L}$ and $\mathrm{S}$, as well as their inhibitor

${ }^{1}$ Department of Vascular and Endovascular Surgery, Klinikum rechts der Isar der Technischen Universitaet Muenchen, Munich, Germany; ${ }^{2}$ Institute of Pathology, Klinikum rechts der Isar der Technischen Universitaet Muenchen, Munich, Germany and ${ }^{3}$ Department of Surgery, Klinikum rechts der Isar der Technischen Universitaet Muenchen, Munich, Germany

${ }^{4}$ These two authors contributed equally to this work.

Correspondence: Dr J Pelisek, Department of Vascular and Endovascular Surgery, Klinikum rechts der Isar der Technischen Universitaet Muenchen, Ismaninger Strasse 22, D-81675 Munich, Germany.

E-mail: J.Pelisek@|rz.tum.de

Received 18 January 2013; revised 24 October 2013; accepted 26 December 2013 
cystatin $\mathrm{C}$, in human AAA samples using quantitative PCR and western blot analyses. The localization of the individual cathepsins was determined by immunohistochemistry (IHC). Furthermore, the corresponding occurrence of these proteases in systemic circulation was evaluated through immunoprecipitation.

\section{MATERIALS AND METHODS}

\section{Patients and tissue collection}

All AAA tissue samples $(n=36)$ were retrieved during open surgical repair. The average age of the study patients was $66 \pm 12$ years. The average maximum AAA diameter derived from the computed tomography scans was $6.2 \pm 1.5 \mathrm{~cm}$. Nonaneurysmal aortic specimens $(n=10)$, which were used as controls, were obtained during autopsy from the Institute of Forensic Medicine. In addition, serum samples were collected from 10 healthy volunteers. Following excision, the tissue samples were divided in two parts. One part was placed in formalin, followed by paraffin embedding, whereas the other part of the specimen was immediately frozen in liquid nitrogen. Fresh frozen samples were used for protein extraction and quantitative western blot analyses; quantitative real-time reverse transcriptase-PCR (RT-PCR) and histological and IHC analyses were performed using the formalin-fixed samples. Serum samples were collected from each AAA patient before surgery and were used for immunoprecipitation.

The study was performed according to the Guidelines of the World Medical Association Declaration of Helsinki. Furthermore, the local ethics committee approved the study, and written informed consent was given by all patients.

\section{Quantitative real-time RT-PCR}

Quantitative real-time RT-PCR was performed using AAA specimens and control aortae that were fixed in formalin and embedded in paraffin and were located adjacent to the IHC samples. RNA was isolated using the High Pure RNA Paraffin Kit according to the manufacturer's instructions (Roche, Mannheim, Germany). The isolated RNA was transcribed into cDNA using the cDNA Synthesis Kit RevertAid (Fermentas, St Leon-Rot, Germany). Quantitative realtime RT-PCR was performed using the SYBR Green fluorescence dye (peqLab, Erlangen, Germany) and the SYBR-Green-Cycler iQ 5 RealTime PCR Detection System (Bio-Rad, Hercules, CA, USA) with the following conditions: initialization step of $5 \mathrm{~min}$ at $95^{\circ} \mathrm{C}$, followed by 40 cycles of denaturation for $10 \mathrm{~s}$ at $95^{\circ} \mathrm{C}$, annealing for $30 \mathrm{~s}$ at $55^{\circ} \mathrm{C}$ (cathepsin L, cathepsin S), $58^{\circ} \mathrm{C}$ (cathepsin B, cystatin C), $60^{\circ} \mathrm{C}$ (cathepsin D, cathepsin $\mathrm{K}$ ) or $62^{\circ} \mathrm{C}(\mathrm{GAPDH})$, and extension for $10 \mathrm{~s}$ at $72^{\circ} \mathrm{C}$. All results were normalized to the expression of glyceraldehyde 3-phosphate dehydrogenase (GAPDH).

The following primer sets were used in this study: forward 5'-TTCTTGCGACTCTTGGGACTTC- $3^{\prime}$ and reverse $5^{\prime}$-TGACGAGG ATGACAGGGAACTA- $3^{\prime}$ for cathepsin B; forward $5^{\prime}$-CCCACACACA CCCACACACTCG- $3^{\prime}$ and reverse ${ }^{\prime}$-CCAGGGAGGGGAAAACCAC AGA-3' for cathepsin D; forward 5'-GGAGCTGACTTCCGCAATC CCG- $3^{\prime}$ and reverse $5^{\prime}$-TGTCTGGCTTCGTTTCGGCAGC- $3^{\prime}$ for cathepsin K; forward $5^{\prime}$-AGGGTCAGTGTGGTTCTTGTTG- $3^{\prime}$ and reverse $5^{\prime}$-TGAGATAAGCCTCCCAGTTTTC- $3^{\prime}$ for cathepsin L; forward $5^{\prime}$-TGTTCACACTTTGCCCTATGAC- $3^{\prime}$ and reverse $5^{\prime}$-AGGGG CTCCATAAGGAAATAAA- $3^{\prime}$ for cathepsin S; forward $5^{\prime}$-GCCTGTGC CTATCACCTCTTAT- $3^{\prime}$ and reverse $5^{\prime}$-CCTTCTCTGTCTGTCTCCT GGT- $3^{\prime}$ for cystatin C; forward $5^{\prime}$-GGGGCTGGCATTGCCCTCAA- $3^{\prime}$ and reverse $5^{\prime}$-GGCTGGTGGTCCAGGGGTCT- $3^{\prime}$ for GAPDH.

\section{Western blot analysis}

Tissue samples were homogenized in liquid nitrogen, suspended in lysis buffer (50 mm Tris- $\mathrm{HCl} \mathrm{pH} 8,150 \mathrm{~mm} \mathrm{NaCl}, 1 \% \mathrm{NP}-40,0.1 \%$ sodium dodecyl sulfate, $0.5 \%$ sodium deoxycholate, $0.02 \%$ sodium azide), and incubated at $4{ }^{\circ} \mathrm{C}$ for $30 \mathrm{~min}$. Cell lysates were collected by centrifugation. The protein concentration of each lysate was determined using the BCA Protein Assay Kit (Pierce Biotechnology, Rockford, IL, USA). Proteins ( $100 \mu \mathrm{g}$ for each sample) were separated by $10 \%$ sodium dodecyl sulfate-polyacrylamide gel electrophoresis and blotted onto a polyvinylidene difluoride membrane. The membrane was blocked in $5 \%$ nonfat milk in Tris-buffered saline $(50 \mathrm{~mm}$ Tris-HCl, pH 7.4, and $150 \mathrm{~mm} \mathrm{NaCl}$ ) for $1 \mathrm{~h}$, followed by consecutive incubation with primary and secondary antibodies (Abs). The blots were developed using a chemiluminescence detection system (SuperSignal West Pico Chemiluminescent Substrate, Thermo Scientific, Waltham, MA, USA). All membranes were reprobed with an antiGAPDH Ab. Protein quantification was performed by comparing the band intensity of each sample with the intensity of the corresponding GAPDH band. The following primary Abs were used: cathepsin B (ab30443, Abcam, Cambridge, UK; dilution 1:1000), cathepsin D (ab6313, Abcam; dilution 1:1000), cathepsin K (ab37259, Abcam; dilution 1:1000), cathepsin L (ab49984, Abcam; dilution 1:1000), cathepsin S (ab18822, Abcam; dilution 1:200), cystatin C (ab33487, Abcam; dilution 1:500) and GAPDH (ab8245, Abcam; dilution 1:5000). The following secondary Abs were used at the listed dilutions: donkey anti-goat IgG (HAF109, R\&D Systems, Minneapolis, MN, USA; dilution 1:1000), goat anti-mouse IgG (TM-001-HR, Thermo Scientific; dilution 1:5000) and goat anti-rabbit IgG (TR-001HR,Thermo Scientific; dilution 1:2500).

\section{Immunoprecipitation}

To determine the levels of cathepsins B, D, K, L and S and cystatin C in the serum of AAA patients and the 10 healthy volunteers, immunoprecipitation was performed using the Catch and Release v2.0 Reversible Immunoprecipitation System, according to the manufacturer's instructions (Millipore Corporation, Billerica, MA, USA). The following Abs were used for detection: cathepsin B (ab30443, Abcam), cathepsin D (ab6313, Abcam), cathepsin K (ab37259, Abcam), cathepsin L (ab49984, Abcam), cathepsin S (ab18822, Abcam) and cystatin C (rabbit polyclonal to cystatin C; ab33487, Abcam). Following immunoprecipitation, the samples underwent western blot analysis as described above.

\section{Histology and IHC}

Histological analysis and IHC were executed on $2-3 \mu \mathrm{m}$ sections of paraffin-fixed samples from AAAs and control aortae. The aortic tissue sections were routinely stained with hematoxylin and eosin and Elastin van Gieson to characterize the morphology of the aortic wall. For antigen retrieval, the tissue sections were either boiled in citric buffer ( $\mathrm{pH}$ 6.0) for several minutes or treated with Protease Type XXIV (Sigma-Aldrich, Steinheim, Germany). Subsequently, the samples were incubated with the appropriate Abs to stain particular cell types: vascular smooth muscle cells (anti-smooth muscle actin, M0635, clone HHF35, Dako, Glostrup, Denmark; dilution 1:200), endothelial cells (anti-von Willebrand factor, M0616, clone F8/86, Dako; dilution 1:500), macrophages/monocytes (anti-CD68, M0814, clone KP1, Dako; dilution 1:2000), T lymphocytes (anti-CD3, A0452, Dako; dilution 1:400) and B lymphocytes (anti-CD20, M0755, clone L26, Dako; dilution 1:500). To detect the expression of the cathepsins and cystatin C, the same Abs that were used for western blot analysis 
were applied. Following primary Ab incubation, von Willebrand Factor and smooth muscle actin were visualized using the APAAP ChemMate Detection Kit (rabbit anti-mouse secondary Ab; Dako) according to the manufacturer's instructions. All other primary Abs were detected and visualized using the peroxidase/DAB ChemMate Detection Kit (Dako). In the case of cathepsin S (primary goat Ab), the secondary $\mathrm{Ab}$ from the kit was replaced with a rabbit anti-goat $\mathrm{Ab}$ (Dako; dilution 1:50).

\section{Statistical analysis}

All statistical analyses were performed using the SPSS for Windows version 17.0. software (SPSS Inc., Chicago, IL, USA). The values of the continuous variables were compared using the nonparametric MannWhitney $U$-test. Significant differences between more than two groups were analyzed using the nonparametric Kruskal-Wallis test. The data are shown in a box plot diagram that represents the median and the 25th and 75th percentiles. All statistical comparisons were two sided in the sense of an exploratory data analysis, using $0.05\left(^{*}\right), 0.01\left(^{* *}\right)$, and $0.001{ }^{(* *)}$ levels of significance.

\section{RESULTS}

\section{Quantitative real-time RT-PCR}

Using quantitative real-time RT-PCR, the mRNA levels of all of the cysteine/aspartic proteases and their inhibitor cystatin $\mathrm{C}$ were detected in the AAA samples and in the control aortae. Following normalization to GAPDH levels, the aortic samples from the control subjects showed significantly increased mRNA expression levels for cathepsin D $(P<0.001)$, cathepsin $\mathrm{K}(P=0.018)$ and cystatin $\mathrm{C}(P=0.002)$ in comparison with the AAA samples (Figure 1). The cathepsin L mRNA level was elevated in the AAA samples compared with the controls, but without postulated significance $(P=0.127)$. Expression of cathepsin B $(P=0.472)$ and cathepsin $\mathrm{S}(P=0.204)$ showed
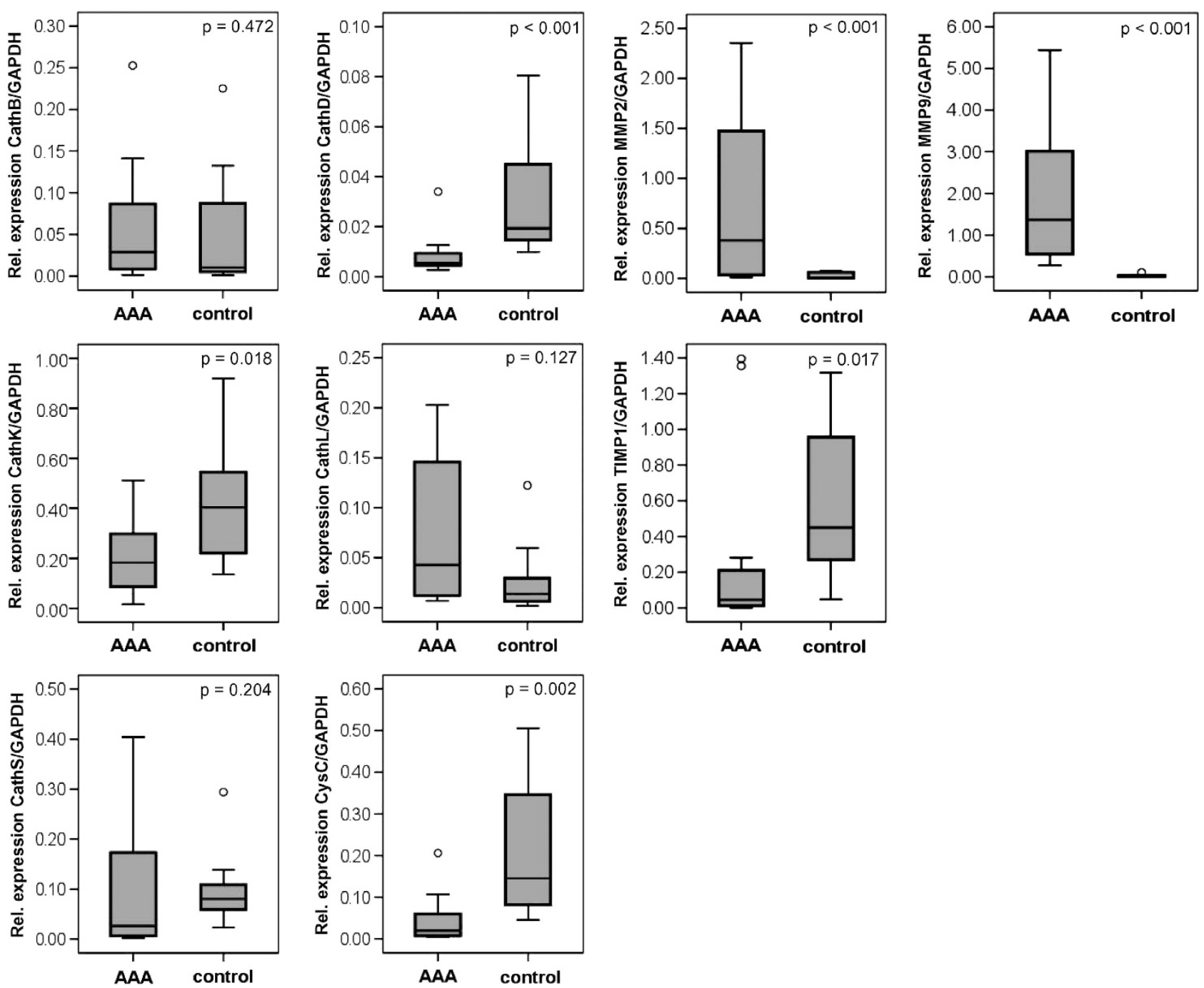

Figure 1 Expression of cathepsins B, D, K, L and S, cystatin C, MMP-2 and -9 and TIMP-1 at the mRNA level in the AAA tissue samples compared with the control healthy aorta samples, as analyzed by quantitative real-time PCR and SYBR green fluorescence dye; the expression levels were normalized to GAPDH. AAA, abdominal aortic aneurysm; Cath, cathepsin; CysC, cystatin C; GAPDH, glyceraldehyde 3-phosphate dehydrogenase; MMP, matrix metalloprotease; TIMP-1, tissue inhibitor of metalloproteinase 1. 
no significant differences between the AAA samples and the control aortic vessels.

Proteases and their functions are closely related to their natural inhibitors, and in the case of cathepsins, cystatin $\mathrm{C}$ plays a major role. Therefore, we also determined the ratio of mRNA expression of the cysteine/aspartic proteases andtheir inhibitor cystatin $\mathrm{C}$ (Figure 2). In this case, the ratio of cathepsin B to cystatin $\mathrm{C}(P=0.021)$ and the ratio of cathepsin L to cystatin C $(P=0.018)$ were significantly elevated in the AAA samples compared with the controls. The cathepsin $\mathrm{K}$ to cystatin $\mathrm{C}$ ratio was also increased in the AAA samples, but without a postulated statistical significance. No significant differences were found between the AAA and control samples for cathepsins $\mathrm{K}, \mathrm{D}$ and $\mathrm{S}$ ( $P=0.286, P=0.979$ and $P=0.517$, respectively). In contrast, the expression levels of MMP-2 and -9 were significantly increased in the AAA samples compared with the control aorta samples $(P<0.001)$, and expression of tissue inhibitor of metalloproteinase 1 (TIMP-1) was also significantly reduced $(P=0.017)$.

In comparing the expression of all cysteine/aspartic proteases with each other, cathepsin $\mathrm{K}$ showed the highest mRNA expression in the AAA samples, as well as in the control aortic vessels, followed by cystatin $\mathrm{C}$ in the healthy aortae (Figures 1 and 2). The expression levels of cathepsins $S$ and $L$ were approximately fivefold lower, and the expression levels of cathepsins B and D were $\sim 10$-fold lower than the expression of cathepsin K. The expression levels of MMP-2 and -9 were 10 - to 100-fold higher than the expression of all of the cysteine/aspartic proteases analyzed in our study.

\section{Western blot analysis}

Following expression analysis at the mRNA level, we determined the expression of cathepsins $\mathrm{B}, \mathrm{D}, \mathrm{K}, \mathrm{L}$ and $\mathrm{S}$ and
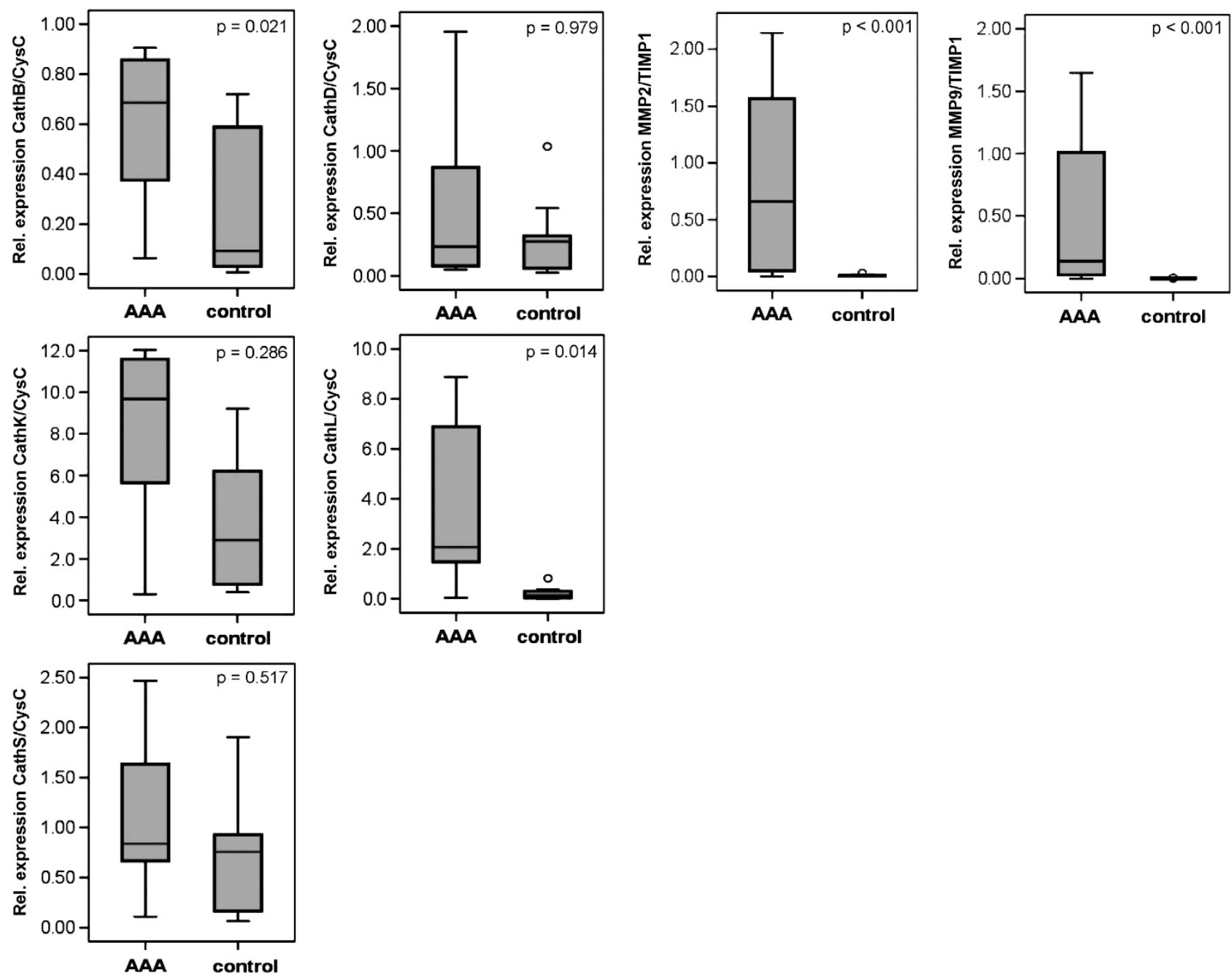

Figure 2 Ratio of the mRNA expression of cathepsins B, D, K, L and S against cystatin C and of MMP-2 and -9 to TIMP-1 in the AAA tissue samples compared with the control healthy aorta samples, as analyzed by quantitative real time PCR and SYBR green fluorescence dye, as shown in Figure 1. AAA, abdominal aortic aneurysm; Cath, cathepsin; CysC, cystatin C; GAPDH, glyceraldehyde 3-phosphate dehydrogenase; MMP, matrix metalloprotease; TIMP-1, tissue inhibitor of metalloproteinase 1. 
a
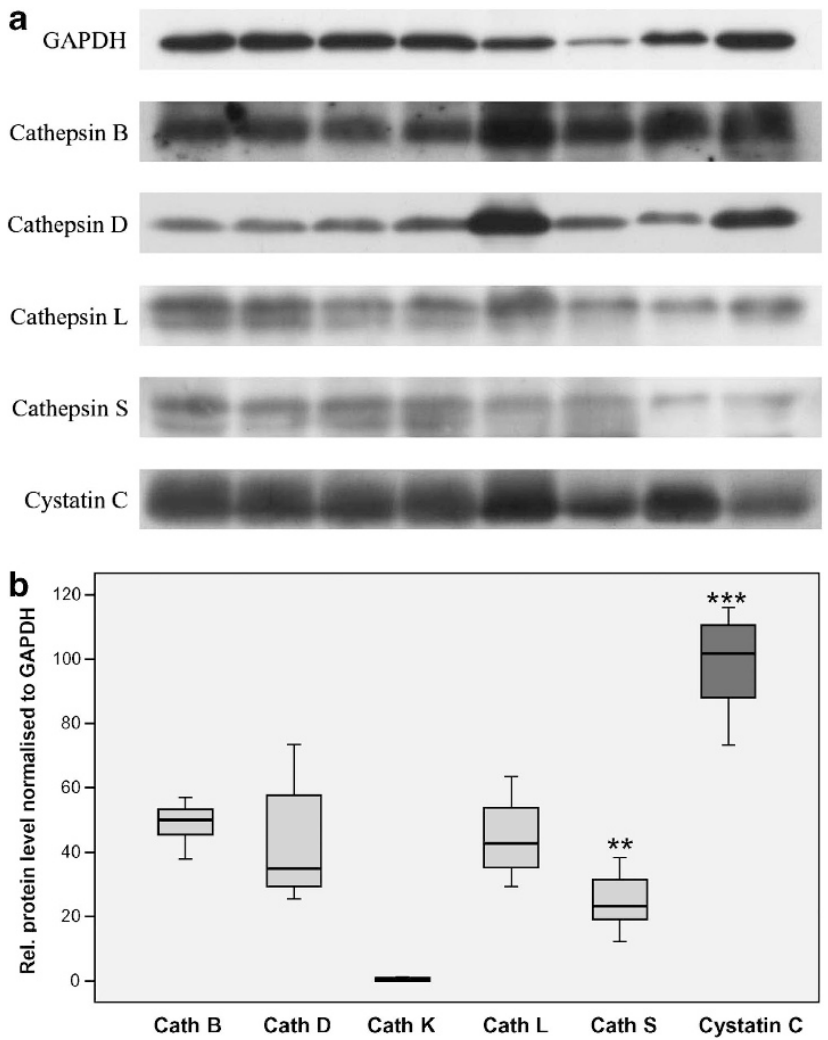

Figure 3 Expression of cathepsins B, D, K, L and S and cystatin C at the protein level in AAA tissue samples, as analyzed by western blot (a), and the summary of quantitative analysis of the band intensity of the single proteinases and their inhibitor cystatin $C(b)$. Fresh tissues samples were used. The intensities of the bands following blotting and chemiluminescence detection were normalized to GAPDH. Expression was adjusted to that of cystatin C, which was set as $100 \%$. AAA, abdominal aortic aneurysm; Cath, cathepsin; GAPDH, glyceraldehyde 3-phosphate dehydrogenase. ${ }^{*} P<0.01, * * * P<0.001$.

cystatin $\mathrm{C}$ in the AAA tissue samples at the protein level using western blot analysis (Figure 3). Unfortunately, no fresh, healthy aorta tissue samples were available. Therefore, we were not able to compare the protein levels of the diseased patients with those of healthy individuals. Interestingly, in contrast to the high expression of cathepsin $\mathrm{K}$ mRNA using quantitative PCR, we were not able to detect the expression of cathepsin K protein in the AAA samples (Figure $4 \mathrm{~b}$ ). The other cysteine/ aspartic proteases were detected at the expected protein size for active enzymes: $31 \mathrm{kDa}$ for cathepsin $\mathrm{B}, 34 \mathrm{kDa}$ for cathepsin $\mathrm{D}, 32 \mathrm{kDa}$ for cathepsin $\mathrm{L}, 30 \mathrm{kDa}$ for cathepsin $\mathrm{S}$ and $16 \mathrm{kDa}$ for cystatin C. An example of the protein bands is shown in Figure $4 \mathrm{a}$, with the exception of cathepsin $\mathrm{K}$ that was not detected; the quantitative expression analyses of the protein levels are shown in Figure 3b. Regarding the amount of protein, the highest concentration was found for cystatin C, followed by cathepsins B, D and L, which had similar levels, and cathepsin S, which was further reduced. These results partially corresponded to the results of the quantitative PCR analysis. The same expression pattern was observed for cathepsins B and D. The protein level of cathepsin L was increased in comparison with the mRNA level, whereas the amount of cathepsin S was decreased compared with its mRNA level. Furthermore, the protein level of cystatin C was significantly higher compared with its mRNA expression. The expression levels of all of the cathepsins lay between $25 \%$ for cathepsin S and 50\% for cathepsin B compared with cystatin $\mathrm{C}$ (comparison of the median values). The only significant differences were observed between cystatin $\mathrm{C}$ and the other cysteine/aspartic proteases $(P=0.001)$. Furthermore, expression of cathepsin $S$ was significantly diminished, compared to cathepsin $\mathrm{B}, \mathrm{D}$, and $\mathrm{L}(P=0.002)$. No expression was detected for cathepsin $\mathrm{K}$.

\section{Immunoprecipitation}

In addition, we also tested whether the cysteine/aspartic proteases analyzed in our study could be detected in the serum of patients with AAA or in healthy individuals. Following immunoprecipitation of the individual cathepsins $\mathrm{B}, \mathrm{D}, \mathrm{K}, \mathrm{L}$ and $\mathrm{S}$ and their inhibitor cystatin $\mathrm{C}$ from the serum samples, western blot analysis was performed as described above. However, with the exception of cathepsin B and cystatin C (Figure 4), no other cathepsins were detected in the serum of the AAA patients or control samples. An example western blot of the detected proteins is shown in Figure $4 \mathrm{a}$, and the quantitative expression analyses of the protein levels are shown in Figure 4b. For cathepsin B, an increase was observed in the AAA patients compared with the healthy controls; however, these differences were not statistically significant. In contrast, significant differences between the study groups were found for cystatin $\mathrm{C}$ and for the cathepsin $\mathrm{B} /$ cystatin $\mathrm{C}$ ratio. The amount of cystatin $C$ in the serum samples of AAA patients was significantly lower $(P=0.001)$, whereas the cathepsin $\mathrm{B} /$ cystatin $\mathrm{C}$ ratio was significantly higher $(P=0.003$; Figure 4$)$.

\section{Histology and IHC}

Finally, to associate the expression of the cathepsins with the cells localized within the AAA, histological and IHC analyses were performed. First, the AAA samples were characterized using hematoxylin and eosin and Elastin van Gieson staining to assess the different features and morphology of the AAA tissue specimens. The main cells detected in the AAA samples, as expected according our previous work, ${ }^{2}$ were smooth muscle cells (SMCs) and inflammatory cells, such as lymphocytes and macrophages. Furthermore, inflammation in the AAA is frequently associated with neovascularization. ${ }^{2,15}$ As expected, markedly increased neovascularization was observed between the media and adventitia and colocalized with the inflammatory cells. In addition, widespread loss of elastin, collagen and SMCs was observed in the AAA samples. Most samples were atherosclerotic, with more or less extended calcified areas.

The IHC analyses of the cysteine/aspartic proteases and their inhibitor cystatin C in the medial SMCs, inflammatory cells and neovessels are summarized in Figure 5. We did not analyze 

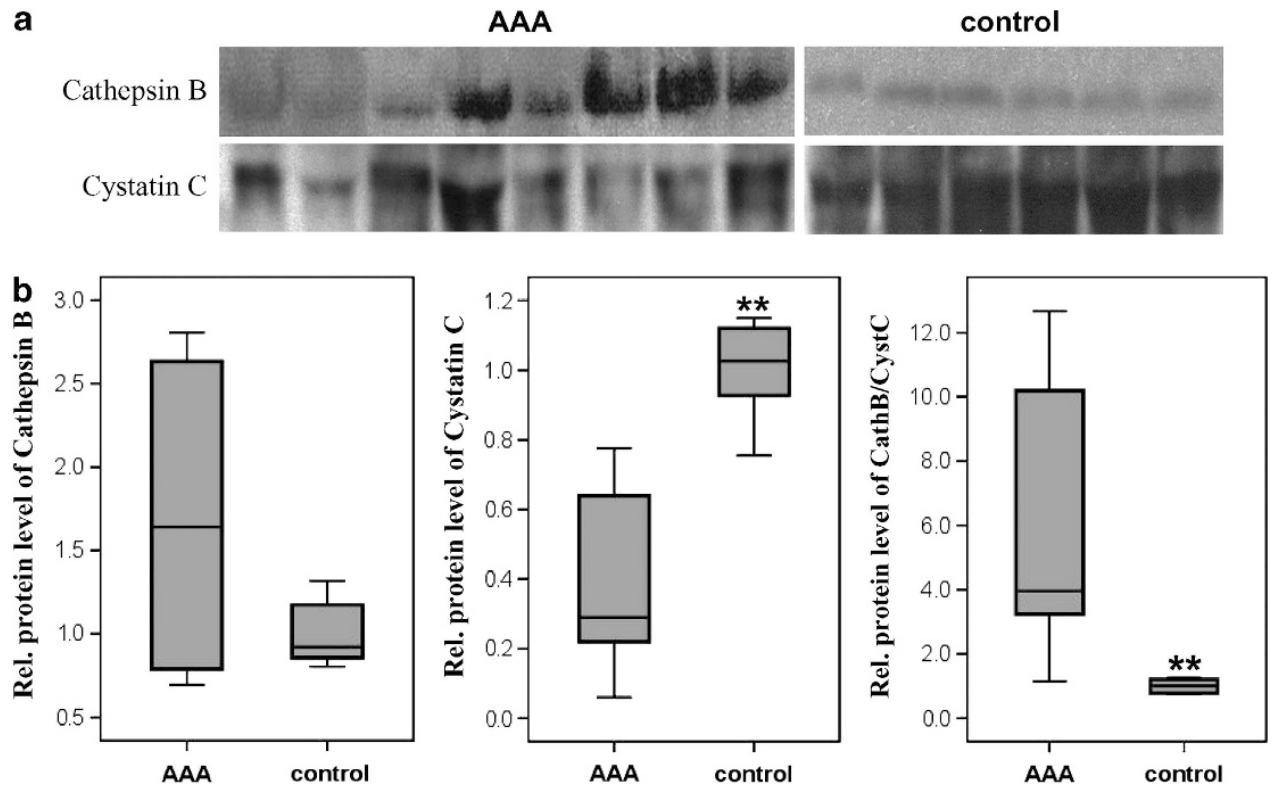

Figure 4 Immunoprecipitation of cathepsin $B$ and cystatin $C$ from the blood serum of AAA patients and healthy volunteers, as analyzed by western blot (a) and the quantitative analysis of the band intensities for cathepsin $\mathrm{B}$ and cystatin $\mathrm{C}$, as well as their ratio (b). AAA, abdominal aortic aneurysm; Cath, cathepsin; CysC, cystatin C. ${ }^{* *} P<0.01$.

endothelial cells on the lumen side because most of our tissues samples did not contain any endothelial cells. SMCs were positive for all investigated proteolytic enzymes and cystatin C (Figure 5a). Macrophages showed the most intensive staining, indicating that these cells exhibited the highest expression of these proteases. Inflammatory infiltrates (lymphocytes) were positive mainly for cathepsin $\mathrm{B}$, followed by cathepsins $\mathrm{D}$ and S. Cathepsins $\mathrm{K}$ and $\mathrm{L}$ and cystatin $\mathrm{C}$ were only weakly detected in these cells (Figure 5b). In the neovessels, cathepsin $\mathrm{B}$ was the most intensive protease observed, followed by cathepsin D (Figure 5c).

\section{DISCUSSION}

Under physiological conditions, the majority of cysteine/ aspartic proteases are localized in intracellular compartments, especially the lysosomes and endosomes where they degrade intracellular and extracellular proteins. ${ }^{13}$ However, under particular conditions, these proteolytic enzymes can also be secreted and are thereby able to act extracellularly. ${ }^{15-18}$ Thus, cathepsins, because of their high proteolytic potential, might play decisive roles in the degradation of the ECM that is essential in AAA pathogenesis. ${ }^{8}$ However, former studies concerning cysteine and aspartic proteases in AAA have left many questions unanswered. ${ }^{15,19-23}$ For this reason, we investigated the relevant cathepsins $(\mathrm{B}, \mathrm{D}, \mathrm{K}, \mathrm{L}$ and $\mathrm{S})$ and their inhibitor cystatin $\mathrm{C}$ in AAA samples and healthy aortae.

All cysteine/aspartic proteases analyzed in our study were detected in the AAA samples, as well as in the healthy aortae. Interestingly, in contrast to the study of Abdul-Hussien et al. ${ }^{21}$ our results did not show the elevated expression of these proteases in the AAA samples in comparison with the nonaneurysmatic aorta samples. Only cathepsin L expression at the mRNA level was increased at an absolute level, but without postulated statistical significance. A possible reason for this discrepancy might be that our control group was older than that used in the work of Abdul-Hussien et al., ${ }^{21}$ who does not mention the exact age of the control subjects. The average age of our control group was $61 \pm 6$ years, and our control group was matched with the age of our AAA patients $(P=0.064)$. In most cases, our control subjects possessed small atherosclerotic changes in the aortic vessel wall, and the intima was enlarged in all cases; it is worth noting that atherosclerosis has already been shown to increase the expression of the cysteine/aspartic proteases. ${ }^{13,15,24,25}$

Because the activities of most of the proteolytic enzymes are closely related to the presence of their inhibitors, the expression level of the cysteine/aspartic proteases alone does not reveal the true proteolytic activity. Therefore, we also calculated the ratio of the proteases to cystatin C. First, we were able to show that cystatin $\mathrm{C}$ expression was significantly reduced in the AAA samples compared with the normal aorta samples. The reduced expression of this inhibitor might be caused by the inflammation processes that are characteristic of aneurysm. ${ }^{26}$ Warfel $e$ e al. ${ }^{27}$ described the downregulation of cystatin C expression following stimulation of monocytes and macrophages. Furthermore, the reduction of cystatin C protein in the AAA samples has also been described by Abdul-Hussien et al., ${ }^{21}$ Abisi et al. ${ }^{22}$ and Sukhova and Shi. ${ }^{28}$ Thus, our results were in agreement with these findings. Regarding the cathepsin/cystatin C ratio based on the mRNA expression, cathepsins B and L showed a significantly elevated 

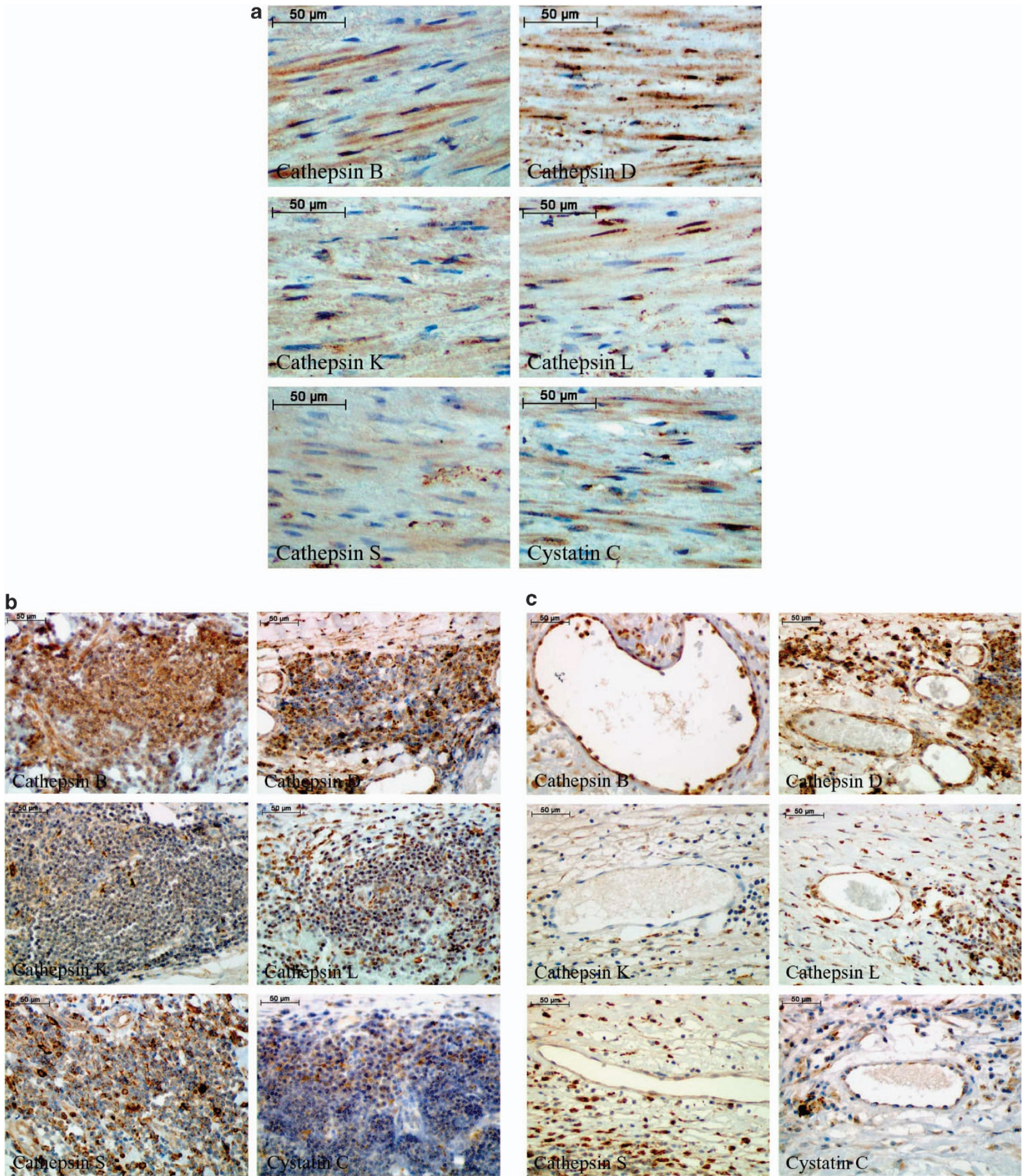

Figure 5 Selective immunohistochemical staining of cathepsins B, D, K, L and S and cystatin C in smooth muscle cells (a), inflammatory cells (lymphocytes) (b) and neovessels (c) within the human abdominal aortic aneurysm (AAA) tissues samples. Cathepsin and cystatin $\mathrm{C}$ staining is brown, and the cells are counterstained with hematoxylin and eosin, shown in blue.

transcription activity in the AAA samples compared with the healthy aortae.

The results from the PCR analysis were partially confirmed using western blot analysis; however, we were not able to detect cathepsin K. Some differences between the mRNA and protein levels were observed for cathepsins $\mathrm{L}$ and $\mathrm{S}$ and cystatin C. However, these discrepancies are not surprising. There are at least two possible reasons for such variability. ${ }^{29}$ 
First, the post-transcriptional mechanisms involved in transforming the mRNA into protein are regulated by different pathways. Thus, the control of the mRNA and protein levels could be independent from each other. ${ }^{29}$ Second, the half-life of the proteins may significantly differ from that of the mRNA. ${ }^{30}$ For example, Gygi et al. ${ }^{31}$ found that even similar mRNA expression levels might be accompanied by an up to 20 -fold difference in protein abundance and vice versa. Furthermore, Lichtinghagen et $a .^{32}$ found no significant relationship between the mRNA and protein levels for the MMP-2 and -9 proteases and their inhibitor TIMP-1.

Regarding the inability to detect cathepsin $\mathrm{K}$, we suppose that one reason for this discrepancy might be the instability of the antibody used in our study. However, it should be mentioned that we tested two different antibodies, but the results were similar (data not shown). Another reason might be the instability of cathepsin $\mathrm{K}$ protein in the extracellular space. However, we have not found any relevant references to support this claim. Therefore, we are not able to explain this discrepancy. Cathepsins B, D, L and S were all detected at the molecular mass corresponding to their respective active forms, which highlights the possible roles of the cathepsins in the pathogenesis of AAA. Thus far, serum analysis of AAA patients has been performed for cathepsins $\mathrm{B}, \mathrm{D}$ and $\mathrm{L}$ and cystatin C. ${ }^{20,23-26,31}$ In contrast to these former studies, we were only able to detect cathepsin B and cystatin C. This could be because of the low sensitivity of the immunoprecipitation or the low occurrence of the other cysteine/aspartic proteases in the blood of our study group. However, cathepsin B, which was the only cathepsin detected in the serum, also had the highest detection level in our western blot analysis. In both the western blot and immunoprecipitation analyses, the active form of cathepsin $\mathrm{B}$, with the molecular mass of $31 \mathrm{kDa}$, was detected. Thus, cathepsin $\mathrm{B}$ might be an interesting protease that should be investigated further, and it would be interesting to evaluate cathepsin $\mathrm{B}$ as a potential biomarker of AAA progression. However, it should be noted that the concentration of cathepsin B in the serum can also be influenced by some carcinomas; ${ }^{33}$ however, this was not the case in our study group.

Finally, to identify cells within the AAA that express the cysteine/aspartic proteases, consecutive immunohistological staining was performed to identify the SMCs, macrophages, lymphocytes and neovessels; these cell types have been previously identified as important sources of proteolytic enzymes, leading to the degradation of the ECM and, consequently, to AAA progression and rupture..$^{2,34,35}$ Furthermore, according to previous investigations, inflammatory cells and SMCs were already identified as important sources of cysteine/aspartic proteases, as well as cystatin C. ${ }^{4,23}$ Our results confirmed the data of Liu et al. ${ }^{13}$ for cathepsins D and L. In addition, weak positive staining was observed for the other cysteine/aspartic proteases and cystatin C. However, lymphocytes, which abundantly invade the aortic vessel wall during the pathogenesis of
AAA, 2,4 have not yet been investigated for cysteine/ aspartic protease expression. Our results demonstrated the expression of cathepsins B, D and S in these cells. Lymphocytes are also able to express various MMPs, as demonstrated in our previous study. ${ }^{2}$ Thus, inflammatory cells may be responsible for further augmentation of inflammation and may also be directly involved in the degradation of ECM, consequently leading to AAA progression and rupture. The results of our study suggest that the main source of cysteine/aspartic proteases in the nonaneurysmatic aortae appears to be the SMCs, according to our IHC investigations, and this hypothesis has been confirmed by others. ${ }^{15}$ In the AAA specimens, we also observed high staining intensity in the macrophages. Following atherosclerotic changes, inflammatory cells, especially monocytes/macrophages and later lymphocytes, accumulate within the vessel wall.,4,14,36 Our results suggest that SMCs may be the main source of cathepsins; this could explain the lower abundance and expression of these proteolytic enzymes in the AAA samples compared with the control aorta samples. These differences might be caused by a partial loss of these cells, which is characteristic of AAA pathogenesis. ${ }^{37,38}$

Regarding the results of the quantitative expression analysis, we have normalized our results to the expression of the GAPDH housekeeping gene, and we calculated the ratio of cathepsins to cystatin C. Regarding GAPDH, even though this housekeeping gene is expressed constitutively and it is recommended for normalization, ${ }^{39,40}$ we cannot exclude the potential changes in its transcription in advanced AAA compared with healthy tissue or between individual AAA specimens. Furthermore, the calculation and usage of the cysteine/aspartic proteases to cystatin $\mathrm{C}$ ratio in our study has some limitations. We do not have any knowledge about the transcriptional regulatory pathways of cystatin $\mathrm{C}$ or its protein stability in comparison with the cathepsins. Furthermore, our study group was quite small; therefore, we cannot exclude bias caused by our assumptions. However, it is worth noting that our results mainly confirmed the data of previously published works. ${ }^{20-22,26}$ Nevertheless, further studies are necessary to determine the functions of the cathepsins in AAA.

In summary, the imbalance between various proteases and their inhibitors is a critical factor that can result in increased proteolytic activity within the aortic wall, leading to vessel wall instability and AAA progression toward rupture. ${ }^{2,9,13}$ In addition to the well-known MMPs, cysteine/aspartic proteases appear to play relevant roles in the pathology of AAA. In addition, the increased expression of cathepsin B could also be detected in the serum of the AAA patients and might serve as a potential biomarker of AAA pathogenesis. Further studies are needed to validate the true role of the cysteine/aspartic proteases in AAA.

\section{CONFLICT OF INTEREST}

The authors declare no conflict of interest. 
1 Eckstein H, Böckler D, Flessenkämper I, Schmitz-Rixen T, Debus S, Lang W. Ultrasonographic screening for the detection of abdominal aortic aneurysms. Dtsch Arztebl Int 2009; 106: 657-663.

2 Reeps C, Pelisek J, Seidl S, Schuster T, Zimmermann A, Kuehnl A et al. Inflammatory infiltrates and neovessels are relevant sources of MMPs in abdominal aortic aneurysm wall. Pathobiology 2009; 76: 243-252.

3 Heider P, Wolf O, Reeps C, Hanke M, Zimmermann A, Berger $\mathrm{H}$ et al. Aneurysms and dissections of the thoracal and abdominal aorta. Chirurg 2007; 78: 600, 602-606, 608-610.

4 Shimizu K, Mitchell RN, Libby P. Inflammation and cellular immune responses in abdominal aortic aneurysms. Arterioscler Thromb Vasc Biol 2006; 26: 987-994.

5 Brewster DC, Cronenwett JL, Hallett JW, Johnston KW, Krupski WC, Matsumura JS. Guidelines for the treatment of abdominal aortic aneurysms. Report of a subcommittee of the Joint Council of the American Association for Vascular Surgery and Society for Vascular Surgery. J Vasc Surg 2003; 37: 1106-1117.

6 Hallin A, Bergqvist D, Holmberg L. Literature review of surgical management of abdominal aortic aneurysm. Eur J Vasc Endovasc Surg 2001; 22: 197-204.

7 Collin J. UK small aneurysms trial. Lancet 1999; 353: 407-408.

8 Krettek A, Sukhova GK, Libby P. Elastogenesis in human arterial disease: a role for macrophages in disordered elastin synthesis. Arterioscler Thromb Vasc Biol 2003; 23: 582-587.

9 Newby AC. Dual role of matrix metalloproteinases (matrixins) in intimal thickening and atherosclerotic plaque rupture. Physiol Rev 2005; 85: 1-31.

10 Keeling WB, Armstrong PA, Stone PA, Bandyk DF, Shames ML. An overview of matrix metalloproteinases in the pathogenesis and treatment of abdominal aortic aneurysms. Vasc Endovascular Surg 2005; 39: 457-464.

11 Ailawadi G, Eliason JL, Upchurch GR. Current concepts in the pathogenesis of abdominal aortic aneurysm. J Vasc Surg 2003; 38: 584-588.

12 Lutgens SPM, Cleutjens KBJM, Daemen MJAP, Heeneman S. Cathepsin cysteine proteases in cardiovascular disease. FASEB J 2007; 21: 3029-3041.

13 Liu J, Sukhova GK, Sun J, Xu W, Libby P, Shi G. Lysosomal cysteine proteases in atherosclerosis. Arterioscler Thromb Vasc Biol 2004; 24: 1359-1366.

14 Yasuda Y, Li Z, Greenbaum D, Bogyo M, Weber E, Brömme D et al. a novel and potent elastolytic activity expressed in activated macrophages. $J$ Biol Chem 2004; 279: 36761-36770.

15 Sukhova GK, Shi GP, Simon DI, Chapman HA, Libby P. Expression of the elastolytic cathepsins $\mathrm{S}$ and $\mathrm{K}$ in human atheroma and regulation of their production in smooth muscle cells. J Clin Invest 1998; 102: 576-583.

16 Liaudet-Coopman E, Beaujouin M, Derocq D, Garcia M, Glondu-Lassis M, Laurent-Matha $\mathrm{V}$ et al. Cathepsin D: newly discovered functions of a longstanding aspartic protease in cancer and apoptosis. Cancer Lett 2006; 237: 167-179.

17 Niedergethmann M, Wostbrock B, Sturm JW, Willeke F, Post S, Hildenbrand R. Prognostic impact of cysteine proteases cathepsin B and cathepsin $L$ in pancreatic adenocarcinoma. Pancreas 2004; 29: 204-211.

18 Lindeman JHN, Hanemaaijer R, Mulder A, Dijkstra PD, Szuhai K, Bromme D et al. Cathepsin $\mathrm{K}$ is the principal protease in giant cell tumor of bone. Am J Pathol 2004; 165: 593-600.

19 Sullivan S, Tosetto M, Kevans D, Kevans D, Coss A, Wang L et al. Localization of nuclear cathepsin $\mathrm{L}$ and its association with disease progression and poor outcome in colorectal cancer. Int J Cancer 2009; 125: 54-61.

20 Lindholt JS, Erlandsen EJ, Henneberg EW. Cystatin C deficiency is associated with the progression of small abdominal aortic aneurysms. $\mathrm{Br}$ J Surg 2007; 88: 1472-1475.

21 Abdul-Hussien H, Soekhoe RGV, Weber E, von der Thüsen JH, Kleemann $\mathrm{R}$, Mulder $\mathrm{A}$ et al. Collagen degradation in the abdominal aneurysm: a conspiracy of matrix metalloproteinase and cysteine collagenases. $\mathrm{Am} \mathrm{J}$ Pathol 2007; 170: 809-817.
22 Abisi S, Burnand KG, Waltham M, Humphries J, Taylor PR, Smith A. Cysteine protease activity in the wall of abdominal aortic aneurysms. J Vasc Surg 2007; 46: 1260-1266.

23 Gacko M, Glowiński S. Cathepsin D and cathepsin L activities in aortic aneurysm wall and parietal thrombus. Clin Chem Lab Med 1998; 36: 449-452.

24 Sukhova GK, Zhang Y, Pan JH, Wada Y, Yamamoto T, Naito M et al. Deficiency of cathepsin $\mathrm{S}$ reduces atherosclerosis in $\mathrm{LDL}$ receptor-deficient mice. J Clin Invest 2003; 111: 897-906.

25 Shi GP, Sukhova GK, Grubb A, Ducharme A, Rhode LH, Lee RT et al. Cystatin $C$ deficiency in human atherosclerosis and aortic aneurysms. J Clin Invest 1999; 104: 1191-1197.

26 Chapman HA, Riese RJ, Shi GP. Emerging roles for cysteine proteases in human biology. Annu Rev Physiol 1997; 59: 63-88.

27 Warfel AH, Zucker-Franklin D, Frangione B, Ghiso J. Constitutive secretion of cystatin $\mathrm{C}$ (gamma-trace) by monocytes and macrophages and its downregulation after stimulation. J Exp Med 1987; 166: 1912-1917.

28 Sukhova GK, Shi GP. Do cathepsins play a role in abdominal aortic aneurysm pathogenesis? Ann NY Acad Sci 2006; 1085: 161-169.

29 Greenbaum D, Colangelo C, Williams K, Gerstein M. Comparing protein abundance and mRNA expression levels on a genomic scale. Genome Biol 2003; 4: 117

30 Glickman $\mathrm{MH}$, Ciechanover A. The ubiquitin-proteasome proteolytic pathway: destruction for the sake of construction. Physiol Rev 2002; 82: 373-428.

31 Gygi SP, Rochon Y, Franza BR, Aebersold R. Correlation between protein and mRNA abundance in yeast. Mol Cell Biol 1999; 19: 1720-1730.

32 Lichtinghagen R, Musholt PB, Lein M, Römer A, Rudolph B, Kristiansen G et al. Different mRNA and protein expression of matrix metalloproteinases 2 and 9 and tissue inhibitor of metalloproteinases 1 in benign and malignant prostate tissue. Eur Urol 2002; 42: 398-406.

33 Herszényi L, Farinati F, Cardin R, István G, Molnár LD, Hritz I et al. Tumor marker utility and prognostic relevance of cathepsin B, cathepsin L, urokinase-type plasminogen activator, plasminogen activator inhibitor type-1, CEA and CA 19-9 in colorectal cancer. BMC Cancer 2008; 8: 194.

34 Halloran BG, Baxter BT. Pathogenesis of aneurysms. Semin Vasc Surg. 1995; 8: 85-92.

35 Guo DC, Papke CL, He R, Milewicz DM. Pathogenesis of thoracic and abdominal aortic aneurysms. Ann NY Acad Sci 2006; 1085: 339-352.

36 Reddy VY, Zhang QY, Weiss SJ. Pericellular mobilization of the tissuedestructive cysteine proteinases, cathepsins $\mathrm{B}, \mathrm{L}$, and $\mathrm{S}$, by human monocyte-derived macrophages. Proc Natl Acad Sci USA 1995; 92: 3849-3853.

37 Henderson EL, Geng YJ, Sukhova GK, Whittemore AD, Knox J, Libby P. Death of smooth muscle cells and expression of mediators of apoptosis by T lymphocytes in human abdominal aortic aneurysms. Circulation 1999; 99: 96-104.

38 López-Candales A, Holmes DR, Liao S, Scott MJ, Wickline SA, Thompson RW. Decreased vascular smooth muscle cell density in medial degeneration of human abdominal aortic aneurysms. Am J Pathol 1997; 150: 999-1007.

39 Silver N, Best S, Jiang J, Thein SL. Selection of housekeeping genes for gene expression studies in human reticulocytes using real-time PCR. BMC Mol Biol 2006; 7: 1-9.

40 Riemer AB, Keskin DB, Reinherz EL. Identification and validation of reference genes for expression studies in human keratinocyte cell lines treated with and without interferon- $\gamma$ - a method for qRT-PCR reference gene determination. Exp Dermatol 2012; 21: 625-629.

(c) (1) () $\Theta$ This work is licensed under a Creative Commons Attribution-NonCommercial-NoDerivs 3.0 Unported License. To view a copy of this license, visit http:// creativecommons.org/licenses/by-nc-nd/3.0/ 